$$
\text { "tmcs-zsombori" — 2013/5/24 - 17:02 — page } 103 \text { - \#1 }
$$

\title{
Teaching probability using graph representations
}

\author{
Gabriella Zsombori and SzilÁRd András
}

An idea that is developed and put into action is more important than an idea that exists only as an idea. Buddha

\begin{abstract}
The main objective of this paper is to present an elementary approach to classical probability theory, based on a Van Hiele type framework, using graph representation and counting techniques, highly suitable for teaching in lower and upper secondary schools. The main advantage of this approach is that it is not based on set theoretical, or combinatorial knowledge, hence it is more suitable for beginners and facilitates the transitions from level 0 to level 3. We also mention a few teaching experiences on different levels (lower secondary school, upper secondary school, teacher training, professional development, university students) based on this approach.
\end{abstract}

Key words and phrases: classical probability theory, graph representations, Van Hiele theory.

ZDM Subject Classification: K53,C33.

\section{Introduction}

Although teaching probability has a strong research background (for a brief overview see [7], Chapter 3) the results of research are not yet incorporated into a large set of coherent and professional teaching materials, or into a practice oriented perspective. Teaching probability theory in secondary school, both on lower and upper level, is usually based on combinatorics and a set theoretic framework. According to this approach the basic probability rules are connected to the set

Copyright (C) 2013 by University of Debrecen 


$$
\text { "tmcs-zsombori" — 2013/5/24 - 17:02 — page } 104-\# 2
$$

operations (intersection, union) and the basic probability models refer to some frequently occurring counting situations (permutations, arrangements, combinations). In many cases students' combinatorial techniques are not deepen enough to constitute a solid basis for probability, hence students have to face a lot of difficulties in understanding and solving probability problems. This is basically because they have to use at the same time their intuitive understanding and also the axiomatic construction of probabilistic models. The approach we use basically reduces the role of set operations and models because it deals directly with the underlying counting problems and for these uses graph representations. The idea of using probability trees appears in a wide range of teaching materials, but in most of them (see [8]) it is not clarified the connection between these trees and the classical definition of probability. It is not clarified that the basics of the classical theory can be built by understanding the structure of these trees. In most of the cases these trees are used for illustrating the basic probability rules, after they were introduced. In [8] the connection between the structure of the trees needed for Copymaster 2.1 and 3.1 is not clarified. We detail these in section 3. This approach is recommended to be used at a beginner level for problem solving and also for the understanding of basic probability notions, models and rules. A major advantage of our method is that it is highly suitable for inquiry based learning (IBL) activities. The importance of IBL has been pointed out in [1], [2], [3]. Based on our approach we organized several IBL teaching activities in Romania with five different types of target groups: 13-14 years old lower secondary school students (group A), 15-17 years old upper secondary school students (group B), 19 years old university students (group C), pre-service teachers (group D) and in-service teachers (D):

- 2 professional development courses (Cluj Napoca, 17-23 July and 5-9 September, 2011), 45 participants;

- 1 conference presentation and workshop (Odorheiu Secuiesc, 4-6 November, 2011), 54 participants;

- 1 conference presentation (Levoča, Slovakia, 20 January, 2012);

- 4 workshops with upper secondary school students (Bonţida, Miercurea Ciuc, Cluj Napoca, Odorheiu Secuiesc), approximately 80 students;

- 2 workshops with lower secondary school students (Târgu Mureş and Miercurea Ciuc)

- 2 workshops for university students (Sapientia University, Miercurea Ciuc and Babeş-Bolyai University at Cluj Napoca), approximately 150 students. 


$$
\text { "tmcs-zsombori" — 2013/5/24 - 17:02 — page } 105 \text { — \#3 }
$$

At these activities the participants were working in groups and after a short introductory phase they usually had to solve several complex problems (the division problem, the Monty Hall problem with several doors, the birthday problem, etc.) using graph representations. The feedback from these activities is highly positive at all levels. All the participants reported that they understood the basic formulas, the use of addition and multiplication while most teachers expressed their believes about the applicability of this method.

\section{The Van Hiele levels}

The Van Hiele theory was initially developed for levels of understanding in geometry $([4],[5])$, but it's framework is more general and can be applied as a theoretical framework for modeling the levels of understanding in learning processes. In this section we describe a Van Hiele type framework for teaching and learning probability and we also emphasize a few critical connections with set theory and combinatorics. From this viewpoint our approach based on graph representations has some major advantages: it supports the transition between the first three Van Hiele levels, it can be used without the set theoretical formalism and without the combinatorial background.

First we list the original Van Hiele levels and we highlight a few characteristics of the model (see [4], [5]).

- Level 0: Intuitive, also called the level of visualization or the level of global recognition. At this level geometric objects are recognized based on their appearance and are connected with the use of common language. For example a square is not recognized as a rectangle, etc.

- Level 1: Analysis (and description). At this level objects are recognized due to their properties, but properties are not organized hierarchically. Usually the relations between different objects and categories are not emphasized.

- Level 2: Abstraction (and informal deduction). At this level properties are organized into a hierarchy, relations between different objects, properties and categories are recognized. The argumentation on this level often depends on perception, there exists some kind of reasoning based on motivated steps, but in general complex and formal proofs are not yet constructed.

- Level 3: Deduction (formal deduction). At this level the formal (complete and correct) proofs are used and constructed. 


$$
\text { "tmcs-Zsombori" — 2013/5/24 — 17:02 — page } 106 \text { — \#4 }
$$

- Level 4: Rigor. This is the level where mathematicians work, where the objects are constructed by axiomatic systems (and are independent of their realizations).

Some researchers use a more elaborated model, with several levels (see [6] for the description of the precognitive level). The importance of these levels is given by the following properties of the levels (see [5]):

- fixed sequence property: students can't skip a level;

- adjacency: properties that are intrinsic at one level become extrinsic at the next level (so the transition between levels has to be prepared);

- distinction: each level has it's own language, terminology that is considered correct at one level may be modified at another;

- separation: teachers explaining (clearly and logically) content at level 3 or 4 are not understood (or are misunderstood) by students on inferior levels (this is a consequence of the distinction property).

A lot of misconceptions in students' learning appear because teachers often disregard these properties. In this section we give a brief description of a Van Hiele type description for levels in understanding probability related notions. We use 5 levels, so we do not describe the level of precognition.

Level 0. (intuitive) At this level the probability is not a mathematical concept but it is used on an idiomatic level as a part of the common language. It is mostly related to percents (used also in weather forecasts) and estimations (not exact values), but students usually are not able to give an acceptable explanation for their phrasing (what it means that "The chance of rain is 40 percent"). Moreover a lot of wrong arguments (every event can occur or not, so the odds are 50-50), misconceptions are used.

REMARK 1. At this level most students are functioning at some kind of "betting mode", they are rather guessing than arguing or calculating. As a consequence their answers are mostly rounded values (50\%, 25\%, 70\%, etc.). Moreover their guessing is also related to their affective condition.

REMARK 2. The probability is often connected to lottery, to gambling games hence the probability of an event is related to luck, to fortune, to chance. But these are unpredictable, so the calculative aspect, the underlying structure of the probability is not emphasized. 


$$
\text { "tmcs-zsombori" — 2013/5/24 - 17:02 — page } 107 \text { — \#5 }
$$

Level 1. (analysis, description) At this level the modeling nature of probability is understood, the meaning and the usage of the words are set as a reference to a model. In this context the probability is used for expressing the chance of occurrence of an event or for calculating the odds (however the odds are expressed as the ratio between the number of favorable and the number of unfavorable events). There are used several representations for the same model:

- diagrams with graphs in order to create counting related representations;

- figures where some kind of quantity (time, length, area, measure of angles) is used in order to create measure related representations.

Moreover the equivalency of different representations is emphasized. The comparison of two probabilities appears as a basic action. The estimative nature of results, the connection with chance, luck, emotional condition disappears, students start focusing on the calculative aspects, the understanding of underlying structures.

Level 2. (order, informal deduction) At this level students can operate with events, they can compute the probability of events by decomposing them into several simple events. The basic connections between algebraic manipulations of the probabilities and the manipulations of events are established, it is possible to define concepts like conditional probability, independent events. All these are strictly related to representations of the models (to counting on graphs, to basic operations with sets, to manipulation of geometric objects, etc.). At this level the representations are created by assigning the objects of the representations to the events of the contexts (so there is a real modelling circle which starts from the context).

Level 3. (deduction) The basic properties of probability are independent of the primary models and representations, they are proved using set theoretical framework (event algebra). At this level the probability models can be treated as basic objects and through the problem solving processes students are assigning the real contexts to the existing models (or to an abstract construction made from these models), hence basic probability distributions (discrete and continuous) are developed as models of possible stochastic behavior (this is a kind of inverse modelling circle, students explore real contexts starting from their mathematical models).

Level 4. (rigor) This is the level of formal logic, based on axioms. The basic fact that for the same set of events we can define several probability measures 


$$
\text { "tmcs-zsombori" — 2013/5/24 — 17:02 — page } 108 \text { — \#6 }
$$

is guaranteed by the axioms, hence the paradoxes from the previous levels (such as the Bertrand paradox) can be solved. At this level the concept of distribution, of stochastic variables becomes a basic tool. This is the level where the mathematics of stochastic processes can be developed.

The fixed sequence property, adjacency, distinction and separation are also characteristics of these levels.

REMARK 3. The previous description emphasizes a major problem in most strategies used for teaching probability theory: the set theoretical foundation is suitable only at the $3^{\text {rd }}$ level and needs also the $3^{\text {rd }}$ level of set theory and combinatorics. Without these prerequisites it is impossible to attain the $3^{\text {rd }}$ level in understanding probability. On the other hand in some countries a few elements of probability are in the curriculum for very young students (7-10 years old in Wales and England, see [7], page 172) and this needs a completely new vision.

\section{Some principles of graph representations}

At an intuitive level the probability is related to odds, to chances, hence at this level the representations can be used basically to illustrate the possibilities in a complex situation or the outcomes in an experiment. In this context the assignment of a number (the probability) to a fixed outcome (event, possibility) is necessary in order to describe, to communicate the situation in a concise, compact way. Since every level has its own language, the same words, expressions may have slightly different meanings at different levels and in the same time most of the descriptions may appear as being incomplete or even wrong at superior levels. This is also valid for the representations, hence in this section we use several representations and for each representation we comment on the corresponding levels. Moreover we consider that two representations of a problem are equivalent if they illustrate the same structure of possibilities for the given problem. This equivalency is related to the equivalency of decision trees, but it is not our intention to give a formal definition for this equivalency relation. The presented representations were developed by lower and upper secondary students during our teaching activities. Throughout this paper we use the classical definition of probability as being the ratio between the number of favorable and all possible outcomes. Our effort focuses on emphasizing the fact that we can construct a teaching/learning environment for basic probability rules using this definition and graph representation on the $1^{\text {st }}$ and $2^{\text {nd }}$ Van Hiele level in order to prepare students for the 


$$
\text { "tmcs-Zsombori" — 2013/5/24 - 17:02 — page } 109 \text { - \#7 }
$$

superior levels. We have to point out that most of the problems from classical probability theory can be solved in this framework, but it is not the intention of the present paper to give an exhaustive discussion, we want only to highlight some crucial aspects.

\subsection{Representing a simple event}

To understand the basic principles of the representation we start with the following problem:

Problem 1. What is the probability that tossing a dice once, the result is divisible by 3 ?

Solution. We have 6 possible outcomes and 2 of them are favorable, so using the definition of the probability of a given event (notated by $A$ ) is $P(A)=$ $\frac{2}{6}=\frac{1}{3}$.

We can represent the possible outcomes in several different ways using a tree with one node (in what follows we use the term node for branch vertices in a tree). Some of these representations are illustrated in Figure 1. a)-f). In case a) we illustrated the 6 different outcomes (which are supposed to be equiprobable), and we marked the 2 favorable cases. In this case we can identify the outcomes with the terminal vertices (leaves) or with the edges from the node to the terminal vertices. The root and the node can be identified with experiment itself. This type of representation can be used starting from the first level of the Van Hiele model (and due to the adjacency this should be used also in order to support the transition between level 0 and 1 ). In case $b$ ) the favorable and non-favorable cases are represented by joining the corresponding terminal vertices from a). On this figure the terminal vertices correspond to the event $A$ and to an event $B$, which is realized exactly when $A$ is not, hence the complementer even $\bar{A}$ appears on the representation. It is worth to mention that on this representation the individual edges correspond to the different outcomes. This representation is suitable on level 1 (when we study very simple events) and it is not recommended on the superior levels because in the case of more events it could lead to a rational error in the counting procedure. The representation c) can be obtained from b) by replacing the multiple edges with a single, but weighted edge. In this case the edge and the terminal vertices are representing the events (that can be composed by several outcomes) while the numbers on the edges represent the ratios between 

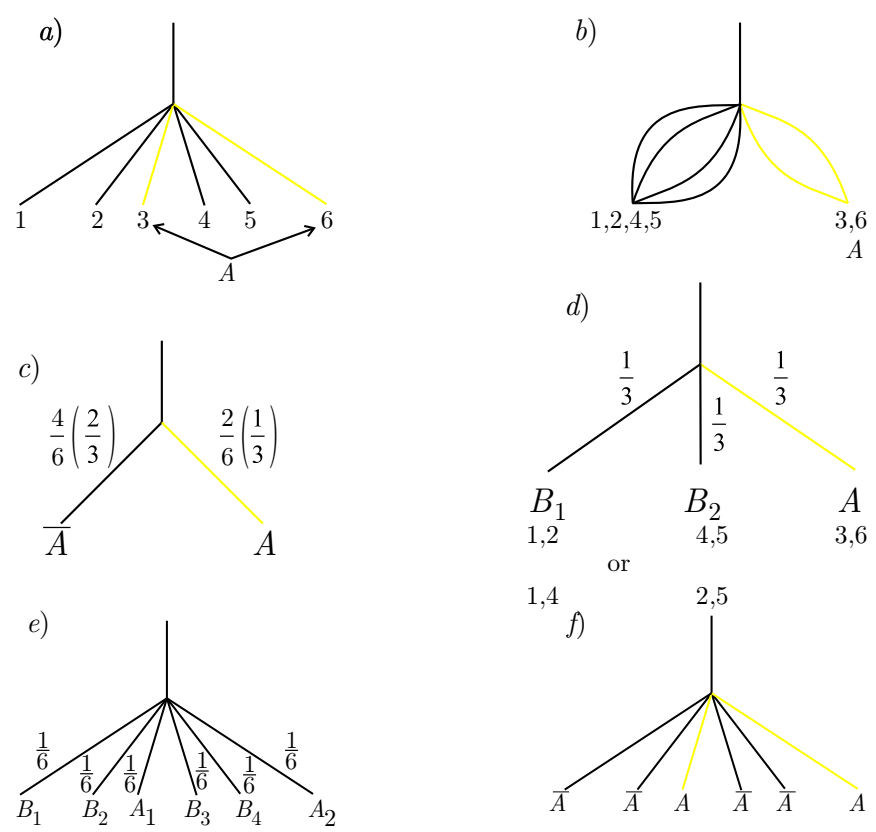

Figure 1. Equivalent representations

the multiplicity of the edge (the number of equiprobable outcomes) and the total number of edges (the number of possible outcomes) starting from the node (note that a tree is a directed graph). This means that in fact these weights are the probabilities corresponding to the events assigned to the edges. This kind of representation is proper to level 2. Figure d) emphasizes two major facts:

- a c) type representation can be transformed into a type a) representation (a tree), if the event $A$ is assigned to some terminal vertices and the edges corresponding to terminal vertices are equally weighted (this is possible if and only if the probability of $A$ is a rational number);

- the same c) type representation can be obtained from several a)- or b)-type representations.

These transformations are needed at level 2 in order to study composite events. It is also important to understand that the events $B_{1}, B_{2}$ on d) can be defined in an arbitrary way under certain constrains (each must contain exactly 2 different outcomes and the outcomes belonging to $B_{1}$ must be different from the outcomes belonging to $B_{2}$ ). 


$$
\text { "tmcs-zsombori" — 2013/5/24 - 17:02 — page } 111 \text { — \#9 }
$$

On level 3 we can use all of the previous representations, but we need a more formal description and notation. For example e) is the same representation as a) but the underlying notations are proper to level 2 (and the superior levels). At this level we have the events $A_{1}, A_{2}, B_{1}, B_{2}, B_{3}, B_{4}$, each of them with probability $\frac{1}{6}$ and the given event is $A=A_{1} \cup A_{2}$ with probability $\frac{1}{6}+\frac{1}{6}=\frac{1}{3}$.

At this level we usually use the representation $\mathrm{c}$ ), where the terminal vertices represent events (outcomes are considered as elementary events) and the numbers assigned to the edges represent the probability of the transition from the event corresponding to the starting node to the event corresponding to the terminal vertex. This can be understood at level 2 after a deeper study of composite events and of more complex situations.

Figure $\mathrm{f}$ ) is also obtained from a) and b) with a different reasoning. In this case to each terminal vertex we assign an even (or a state of the experiment), while the edges corresponds to outcomes. Note that this is somewhat inverse to c) because here we assign the events to the vertices and not the vertices to the events, hence we can have more vertices with the same label. This is related to the viewpoint based on decision trees or flow charts. In this case the constructed tree can be viewed as a decision tree, where the edges corresponds to possible transitions (outcomes or even events if we use weighted edges) while the labels of vertices give the corresponding state of the system, obtained after the chosen transitions. In this view a path from the root to a terminal vertex is a possible sequence of events corresponding to an experiment. Although this kind of representation can be used at level 1 (and the superior levels), it might be helpful to clarify at level 2 the connection between possible representations and also their relationship with set operations (through a set of simple tasks).

As we illustrated even for the simplest problem we have a large variety of equivalent representations but they might cover slightly different reasonings.

REMARK 4. Moreover, there are many other possibilities to use a tree representation. These equivalent representations are necessary when we need to emphasize the equally probable events, see also Remark 12 and are usually not developed by students at the first attempts because in these cases they have to define outcomes that are not defined in the initial context. One of these could be a tree with a node of degree 12 (or 24 , or 30 ) in which all the edges have probability $\frac{1}{12}$ and there are 4 disjoint outcomes that constitute $A$. These 12 cases can be identified with the outcomes of the dice throwing and an imaginary, perfect coin which is tossed (imaginary) at the same time as the cube. So 3(1) means, that 3 appeared on the dice and a head appeared on the coin and 3(2) means that 


$$
\text { "tmcs-zsombori" — 2013/5/24 - 17:02 — page } 112 \text { — \#10 }
$$

3 appeared on the dice, while a tail appeared on the coin. This is illustrated in Figure 2.

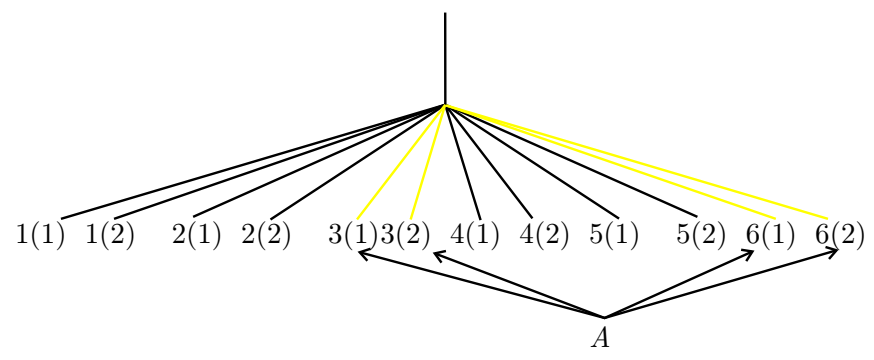

Figure 2. One more equivalent representation

This multitude of representations emphasizes the fact that the equally probable events can be regarded as equivalent events, so in order to calculate the probability of a specific event we can work with any other equivalent event.

It is possible to use a lot of other representations. We can use the unfolded cube, or six squares, each of them representing one face of the cube. All these representations have a common feature: there are 3 times more possible cases than favorable cases.

REMARK 5. In order to connect with level 0 it is recommended to formulate the same properties (on each representation) also using the term odd: The odds for obtaining a number divisible with 3 are $2: 4$ (or $1: 2$ ).

REMARK 6. If we organize IBL activities and we do not provide any structure for how to represent the possibilities, students can develop a multitude of representations. In this situations the role of teacher is to help students to explain all the details of their conception and to compare different representations through solving more problems. Case f) is such an example, which is not usually known and accepted in the literature.

\subsection{Representations for composite events}

If for the representation of an event we have a lot of possibilities, than it is obvious (or common sense) that the choice of a convenient representation can help a lot in understanding more complicated phenomena. Our aim is to show that in almost all the cases that we are facing in teaching probability theory at 


$$
\text { "tmcs-zsombori" — 2013/5/24 — 17:02 — page } 113 \text { — \#11 }
$$

an undergraduate level, we can choose a representation that permits to calculate probability as the ratio between the number of favorable events and the number of all possible events. As a first example consider the following problem:

Problem 2. Let $A$ be the event that tomorrow afternoon is going to be a sunny weather. Let the probability of this event be, $P(A)=\frac{1}{3}$. Meanwhile let $B$ be the event that Peter's bike is out of order and it is going to be repaired by tomorrow afternoon. Let the probability of this event be, $P(B)=\frac{1}{4}$. What is the probability that Peter can ride his bike tomorrow afternoon in a sunny weather?

REMARK 7. We suppose that there is no connection between the weather and the reparation.

Solution. On Figure 3. we visualized these events. One can see the events $A, B$ and their complement events. The probability of these events are

$$
P(A)=\frac{1}{3}, \quad P(\bar{A})=\frac{2}{3}, \quad P(B)=\frac{1}{4}, \quad P(\bar{B})=\frac{3}{4} .
$$

On Figure 5. these graphs are structured differently, so that the choices at each
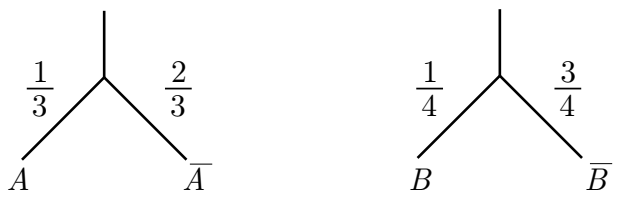

Figure 3. The probability of the events $A$ and $B$

level to have the same probability. As pointed out in Remark 4. this is always possible if we consider some auxiliary (imaginary) events, one with probability $\frac{1}{2}$ coupled with $A$ and two more with probabilities $\frac{1}{3}$ (these events can belong to a random generated number from the interval $[0,1]$ or to a coin and a dice tossing). We note that if the edges starting from a node do not have numbers written on them, it means that they are equally probable.

REMARK 8. For the sake of clarity we detail the second tree on Figure 5. with a description on level 3 . Consider a dice tossing and the events $C_{1}, C_{2}, C_{3}$ corresponding to the following results $\{1,2\},\{3,4\}$ and $\{5,6\}$ respectively. In this way we can decompose the event $\bar{B}$ into three parts having the same probability, 


$$
\text { "tmcs-zsombori" — 2013/5/24 — 17:02 — page } 114 \text { — \#12 }
$$

hence we have the tree on Figure 4., where the original event $B$ can be associated to a terminal vertex (and viceversa) such that all the events associated to the terminal vertices have the same probability. In Figure 5 the three edges to $\bar{B}$

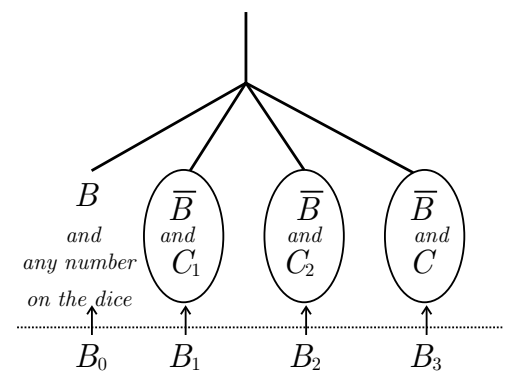

Figure 4. Redifining the events in order to obtain bijection between equally probable events and the terminal vertices

represent the events $C_{1}, C_{2}$ and $C_{3}$, but these are not essential for the solution of the problem, so on lower levels $(0,1,2)$ it is not required to describe them explicitly.
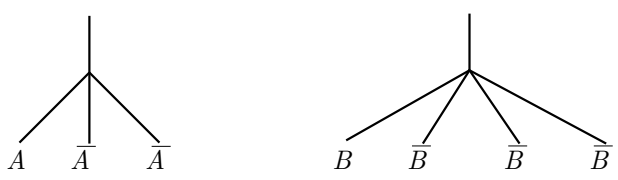

Figure 5. Restructuring the graphs in order to emphasize equally probable choices

If we place to each terminal vertex of the first tree of Figure 5 the root of the second tree (this can be done since there is no connection between the events) and we neglect these roots we obtain the Figure 6 , which reflects both the structure of all possibilities (in the simultaneous analysis of both events) and the weight of these, so in the next step we can simplify the representation in order to obtain Figure 8. This representation can be converted to a more precise one (where the correspondence between vertices and events is one-to-one) if we replace the labels of the terminal vertices with $A \cap B, A \cap \bar{B}, \bar{A} \cap B$ and $\bar{A} \cap \bar{B}$. It is clear that if we want switch to a level 3 description (and notations) on Figure 6 , than we will have events like $A \cap B, A \cap B_{1}=A \cap \bar{B} \cap C_{1}$, etc. Figure 6 . shows that there are 12 equally probable outcomes and only one of them is favorable, so the probability 
that Peter can ride his bike tomorrow afternoon in a sunny weather is $\frac{1}{12}$. This shows that if we use these representations (or some similar representations), we can solve the problem on level 1. If we want to facilitate the transition to the next level we can formalize the counting argument and we can emphasize the set theoretical operation. In fact the problem asks for the probability of $A \cap B$, the intersection of the events $A$ and $B$ (which occurs if and only if both events occur). Hence from the previous argument we have

$$
P(A \cap B)=\frac{1}{12}
$$

On the other hand if we analyze the simplified form of the graph (shown in Figure

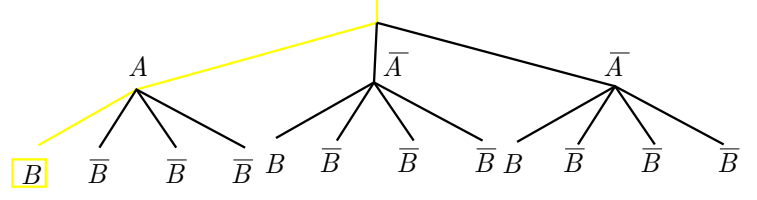

Figure 6. $A$ and $B$ together

7.) or we think about how we calculated the number of outcomes, we can observe that $\frac{1}{12}$ is the product of the probabilities corresponding to the edges belonging to the emphasized path (in this case this is the product of the probabilities of the events $A$ and $B)$.

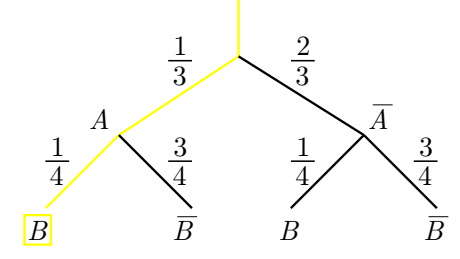

Figure 7. The simplified version of the graph from Figure 6.

REMARK 9. The previous figures (and the solution of this problem) illustrate a few important steps in studying composite events:

- constructing the detailed graph (where all the choices have the same probability, hence the calculation of the probability resumes to a counting argument); 
- joining the graphs corresponding to different events (constructing the levels);

- simplifying the detailed graph of the composite event.

These steps are crucial in order to understand the background of the algebraic operations we are performing with probabilities.

We can exchange the levels corresponding to the events $A$ and $B$ in the construction of the graph in Figure 6. This is shown on Figure 8. (the detailed version) and on Figure 9. (the simplified version). From this figure we obtain $P(B \cap A)=\frac{1}{12}$.

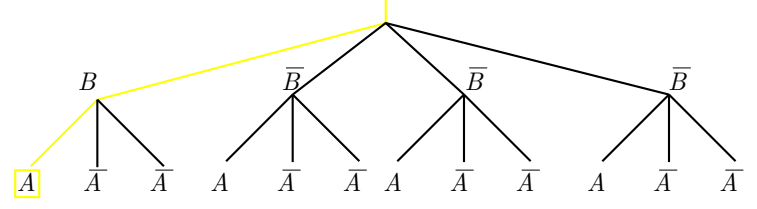

Figure 8. Switching the levels in Figure 6.

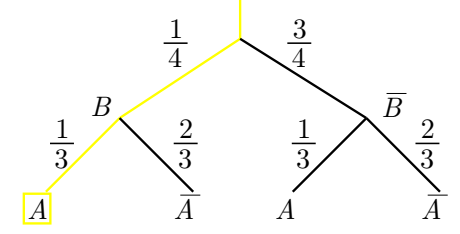

Figure 9. The simplified version of the graph from Figure 8.

\subsection{Representation for conditional probability}

We can use the previous technique even if the events are connected, so we have to different trees to the terminal vertices of the first tree. In this subsection we don't comment on the different types of representations for each figure separately. All these figures are using representations suitable starting from level 1. Of course on superior levels they might need more detailed explanations. 
Problem 3. We have two bags: in the first there are 4 red and 1 black balls, while in the second one there are 2 red and 3 black balls (see Figure 10.). We throw a dice once. If the number shown on the dice is divisible by three, we pull out a ball from the first bag, otherwise we do the same, but from the second bag. What is the probability that the extracted ball is black?

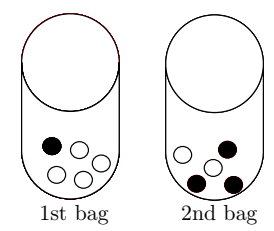

Figure 10. Bags and balls

Solution. The events from the problem are illustrated on Figure 11. Using this figure, the number of equally probable outcomes is 30 , while the number of favorable outcomes is 14 , hence the desired probability is $\frac{14}{30}$. On the simplified

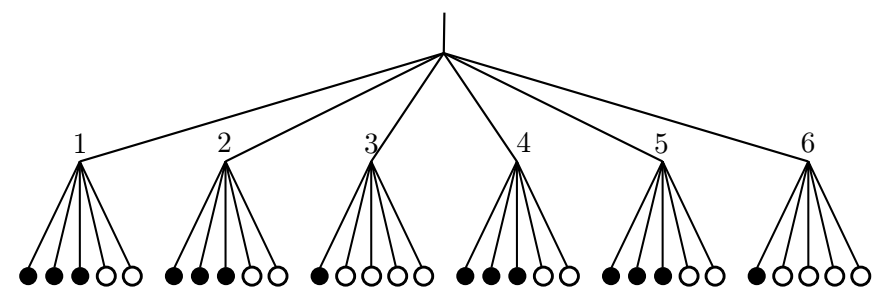

Figure 11. The detailed graph for the extraction of the ball

graph (Figure 12.) we can observe that

$$
\frac{14}{30}=\frac{2}{6} \cdot \frac{1}{5}+\frac{4}{6} \cdot \frac{3}{5}
$$

REMARK 10. On Figure 12. we can observe that the probability of the black ball's extraction is different in the two cases. This motivates the introduction of the conditional probability. If we denote the apparition of 3 or 6 on the dice with $A$ and the extraction of a black ball with $B$, then the numbers $\frac{1}{5}$ and $\frac{3}{5}$ on the edges are in fact conditional probabilities: 


$$
\text { "tmcs-zsombori" — 2013/5/24 — 17:02 — page } 118 \text { — \#16 }
$$

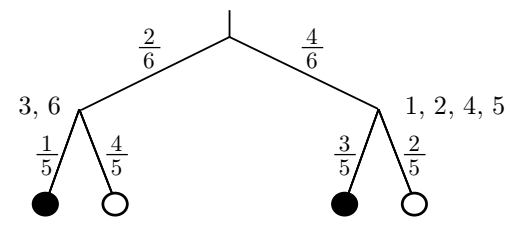

Figure 12. The simplified graph for the extraction of the ball

- $\frac{1}{5}$ is the probability of the event $B$, if we know that $A$ already occurred; to reflect this in our notations we use the symbol $P(B \mid A)$;

- $\frac{3}{5}$ is the probability of the event $B$, if we know that $\bar{A}$ already occurred; we denote this by $P(B \mid \bar{A})$.

Generally the notation $P(X \mid Y)$ stands for the conditional probability of the event $X$ with respect to the event $Y$ (and it is the probability of the event $X$, if the event $Y$ already occurred). Using this notation and the observations we made previously (at problem 2. and 3.) we can formulate the following properties:

$$
P(A \cap B)=P(A) \cdot P(B \mid A), \quad \text { and } \quad P(B \cap A)=P(B) \cdot P(A \mid B) .
$$

From these we deduce:

$$
P(A \cap B)=P(B \cap A)=P(A) \cdot P(B \mid A)=P(B) \cdot P(A \mid B) .
$$

In problem 2. we had

$$
P(B \mid A)=\frac{1}{4}=P(B) .
$$

This means that the events $A$ and $B$ are independent. In problem 3. the events $A$ and $B$ are not independent. In the same way using the graph representation we can check that for two independent events $A$ and $B$ we have:

$$
P(A \cap B)=P(A) \cdot P(B) .
$$

REMARK 11. Using the graph representations we can introduce and study all basic notions from classical probability theory (the probability of a reunion, law of total probability, Bayes' theorem, classical probability models).

REMARK 12 . We can guarantee equally probable outcomes only if the nodes situated on the same level have the same degree, so all the detailed graphs must have this property. The counting argument applies exclusively to these graphs. In order to make it happen, we might need the following restructuring: 

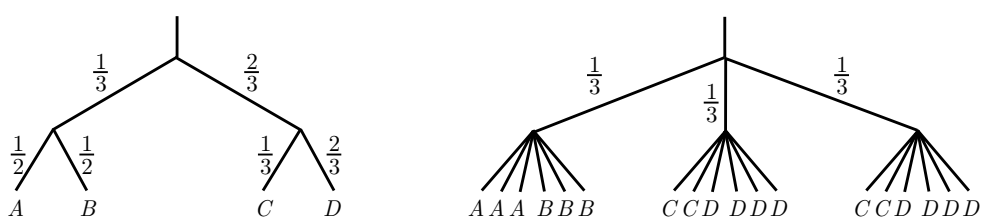

Figure 13. Emphasizing the equally probable choices

- at each level we have to find a number $p_{0}$ with the property that all the probabilities at this level are natural multiples of $p_{0}$ (see Figure 13.);

- if some edge of the graph is joining nodes from nonconsecutive levels, then we have to add new nodes (see Figure 14.).
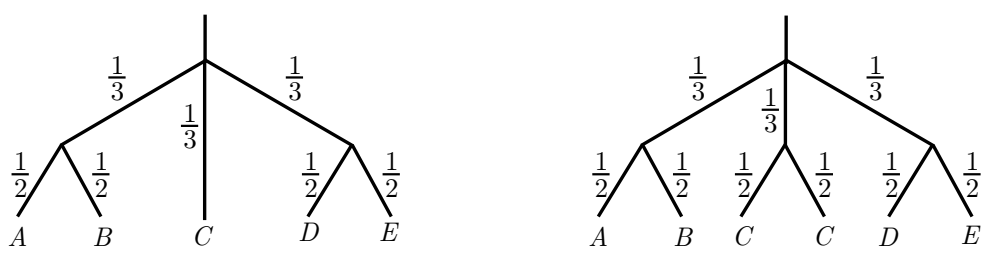

Figure 14. Adding a new node

By inverting the above steps we can obtain the simplified graphs (which are weighted graphs).

REMARK 13. For the illustration of main ideas and difficulties we used simple exercises without any connection. This was motivated by practical reasons, we wanted to emphasize (specially for practicing teachers) that the main difficulties can (and should be) addressed and clarified using simple tasks first and also the fact that in an implementation phase these difficulties needs different teaching activities.

\section{Final remarks}

- The presented approach proves that all the concepts, properties, models from the undergraduate probability curricula can be introduced using graph representations. This makes possible to begin at Van Hiele level 0, to avoid the set theoretical framework at the beginning (hence we avoid overlapping the 
"tmcs-zsombori" — 2013/5/24 - 17:02 — page 120 — \#18

difficulties from set theory, combinatorics and probability) and go through level 1 and 2 .

- The description of Van Hiele levels gives a partial answer to most of the teaching difficulties and student misconceptions that appear due to the fact that the level used by teachers is not the same as the level of students.

- In order to avoid set theoretical approach based on the simultaneous use of algebra (for calculating probabilities) and event algebra (for decomposing events) at the beginning, for didactic reasons, we used a slightly simplified notation on the graphs. Starting from Figure 5., instead of the notation used on Figure 1./e), we denoted by the same letter $X$ the equally probable subevents resulted from the decomposition of an event $X$. As we pointed out in Remark 4. this decomposition is always possible with the use of auxiliary experiments and events. On the other hand, if we use the notation from Figure 1./e), we can easily connect the counting arguments to the set theoretical approach (and viceversa). From this viewpoint, our approach has a great advantage: we do not need to detail the decomposition of an event into a given number of equally probable sub-events, while in the set theoretical approach this would be necessary. For example how to decompose the coin tossing into 30 equally probable sub-events.

- The graph based approach with equally probable outcomes can not be used if the probability of an event is not a rational number (because the degree of a node multiplied by the probability of an edge starting from this node is 1), but

- if we use weighted trees, we can use irrational weights too and we omit the complete proof of this fact (based on limits) if it is not required in the context, we mention that the same procedure is applied for other notions too (like the area of a rectangle having irrational side lengths) in the school practice.

- before the $3^{\text {rd }}$ Van Hiele level the use of irrational numbers is not necessary, teaching probability theory to lower secondary school students usually does not imply events with irrational probability;

- facilitates inquiry based learning starting from the 0 Van Hiele level (without needing prerequisites of higher level), students can easily establish all the basic relations by themselves, simply by reading the graphs;

- With groups $\mathrm{A}$ and $\mathrm{C}$ we had a longer sequence of activities (more than 20 hours with each group) where the main aim was to introduce the classical 


$$
\text { "tmcs-zsombori" — 2013/5/24 — 17:02 — page } 121 \text { — \#19 }
$$

probability using this framework and IBL activities. The details of these activities do not constitute the aim of this paper, we only emphasized the main crucial ideas which can be used in developing further teaching materials.

\section{Acknowledgements}

This paper is based on the work within the project Primas. Coordination: University of Education, Freiburg. Partners: University of Genève, Freudenthal Institute, University of Nottingham, University of Jaen, Konstantin the Philosopher University in Nitra, University of Szeged, Cyprus University of Technology, University of Malta, Roskilde University, University of Manchester, Babeş-Bolyai University, Sør-Trøndelag University College. The authors wish to thank their students and colleagues attending the training course organized by the BabesBolyai University in the framework of the FP7 project PRIMAS ${ }^{1}$, they were partially supported by the SimpleX Association from Miercurea Ciuc. Both authors wish to express a deep sense of gratitude to the anonymous referees for their valuable comments, which contributed crucially to the improvement of the paper.

\section{References}

[1] Cs. Csíkos, Problémaalapú tanulás és matematikai nevelés, Iskolakultúra, 2010/12.

[2] H. Wallberg-Henriksson, V. Hemmo, P. Csermely, M. Rocard, D. Jorde and D. Lenzen, Science education now: a renewed pedagogy for the future of Europe, http://ec.europa.eu/research/science-society/document_library/pdf_06/ report-rocard-on-science-education_en.pdf.

[3] S. Laursen, M. M.-L. Hassi, M. Kogan, A.-B. Hunter and T. Weston, Evaluation of the IBL Mathematics Project: Student and Instructor Outcomes of Inquiry-Based Learning in College Mathematics, Colorado University, April 2011.

[4] P. M. Van Hiele, Development and learning process, Acta Paedogogica Ultrajectina (1959), 1-31, Groningen: J. B. Wolters.

[5] Z. Usiskin, Van Hiele levels and achievment in secondary school geometry, The University of Chicago, 1982.

[6] D. Clements and M. Battista, Geometry and spatial reasoning, in: Handbook of Research on Mathematics Teaching and Learning, (D. Grouws, ed.), New York, NY: Macmillan, 1992, 420-464.

${ }^{1}$ Promoting inquiry in Mathematics and science education across Europe, Grant Agreement No. 244380 


$$
\text { "tmcs-zsombori" — 2013/5/24 - 17:02 — page } 122 \text { — \#20 }
$$

[7] Exploring Probability in School, (G. A. Jones, ed.), Springer, 2005.

[8] http://www2.nzmaths.co.nz/statistics/Probability/Probtree.aspx.

GABRIELLA ZSOMBORI

SAPIENTIA UNIVERSITY

MIERCUREA CIUC

ROMANIA

E-mail: zsomborigabriella@gmail.com

SZILÁRD ANDRÁS

BABES-BOLYAI UNIVERSITY

CLUJ NAPOCA

ROMANIA

E-mail: andraszk@yahoo.com

(Received September, 2012) 